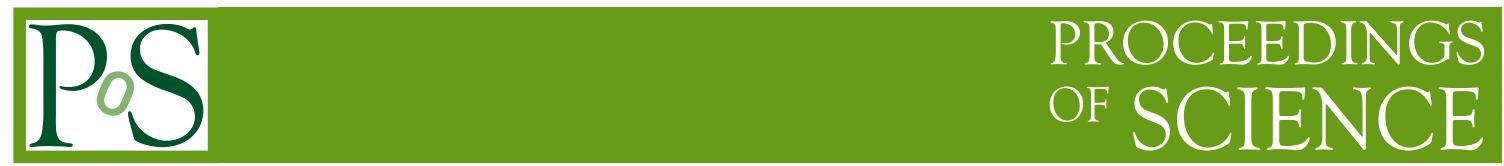

\title{
Studies of charmonium-like particles at Belle II
}

\author{
Rashmi Dhamija ${ }^{a, *}$ \\ ${ }^{a}$ Indian Institute of Technology, Hyderabad \\ Telangana, India \\ E-mail: rshmiphysicist@gmail.com
}

The Belle II experiment has accumulated data corresponding to $213 \mathrm{fb}^{-1}$ of integrated luminosity in the past two years. Belle II plans to accumulate $50 \mathrm{ab}^{-1}$, which are expected to bring a large impact to the field of hadron spectroscopy. We present here the analysis of $B \rightarrow K J / \psi \pi^{+} \pi^{-}$ and $B \rightarrow K \psi(2 S)$ decays using $62.8 \mathrm{fb}^{-1}$ at Belle II. We find evidence of $X(3872) \rightarrow J / \psi \pi^{+} \pi^{-}$ decays with a signal yield of $14.4 \pm 4.6$ and a statistical significance of $4.6 \sigma$. We also present preliminary results of $e^{+} e^{-} \rightarrow \pi^{+} \pi^{-} J / \psi$ via ISR using $37.8 \mathrm{fb}^{-1}$.

*** The European Physical Society Conference on High Energy Physics (EPS-HEP2021), ***

*** 26-30 July $2021 * * *$

*** Online conference, jointly organized by Universität Hamburg and the research center DESY ***

${ }^{1}$ On behalf of the Belle II collaboration.

* Speaker 


\section{Introduction}

The Gell-Mann, Nee'man and Zweig idea, known as the Constituent Quark Model, [1][2] describes the known hadrons, namely the two- (mesons) or three-quark (baryons) combinations. One such possible epitome is quarkonium which is a bound state of heavy quark $(b, c)$ with its antiquark. This system is explained by various effective models based on quantum chromodynamics (QCD), the theory of strong interaction. However, the QCD-based effective models predict the existence of complex structures known as exotic states involving more than three quarks, which were observed with the discovery of $X(3872)^{1}$ at Belle [3] in 2003 and confirmed by other experiments [4-7]. More than 30 exotic candidate states have been observed after the discovery of $X(3872)$, mostly found in the charmonium sector via $B$ decays and direct production.

The $B$-factories have made rich contributions to quarkonium spectroscopy. However, some interesting signatures such as $Z_{c}(3900), Z_{c}(4430)$, etc. are not yet established. More data are needed to understand and unravel the situation. Belle II is expected to help improve our understanding in this sector.

\section{SuperKEKB and Belle II}

Belle II experiment [9] is located at the SuperKEKB asymmetric $e^{+}-e^{-}$collider at KEK in Tsukuba, Japan. SuperKEKB is designed to reach a peak luminosity $6 \times 10^{35} \mathrm{~cm}^{-2} \mathrm{~s}^{-1}$, about 30 times higher than that of its predecessor KEKB. The instantaneous luminosity achieved so far is $3.1 \times 10^{34} \mathrm{~cm}^{-2} \mathrm{~s}^{-1}$. The goal of the Belle II experiment is to accumulate a data set of $50 \mathrm{ab}^{-1}$, 50 times larger than that of its predecessor Belle. As compared to Belle, Belle II can provide better vertexing resolution and improved momentum resolution. Figure 1 shows sketches of the SuperKEKB and the Belle II detector.
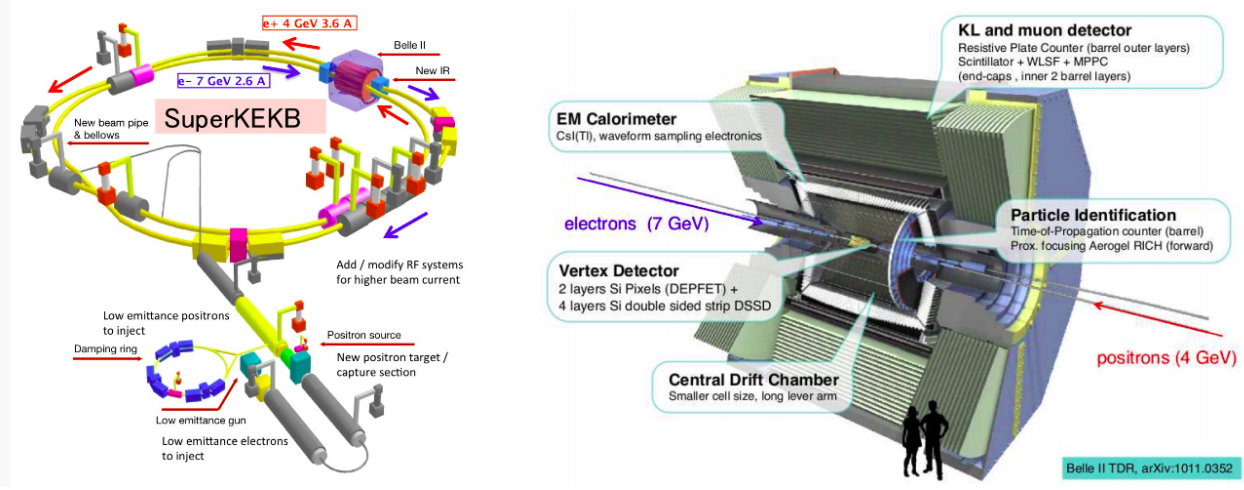

Figure 1: Overview of SuperKEKB and Belle II detector.

\section{3. $X(3872)$ studies at Belle II}

The $X(3872)$ was first discovered in $B \rightarrow K J / \psi \pi^{+} \pi^{-}$decays. It has been widely studied in various decay modes. However, the complete nature of this state is not fully clear yet.

\footnotetext{
${ }^{1}$ It is called $\chi_{c 1}(3872)$ in PDG.
} 
The early reconstruction for $X(3872)$ at Belle II is done by studying $B \rightarrow K J / \psi \pi^{+} \pi^{-}$decays and using a sample corresponding to $62.8 \mathrm{fb}^{-1}$ collected at the $\Upsilon(4 \mathrm{~S})$ resonance. Charged particles are required to originate from the interaction point by restricting their azimuthal (with respect to the beam axis) and longitudinal displacement. $K_{s}^{0}$ mesons are reconstructed by combining two oppositely charged pion candidates, subjecting them to a vertex fit, and constraining the resulting $K_{s}^{0}$ candidate mass $m\left(\pi^{+} \pi^{-}\right)$to be within 490 and $506 \mathrm{MeV} / \mathrm{c}^{2} . J / \psi$ mesons are reconstructed from a pair of oppositely charged electron or muon tracks. After requiring the $J / \psi$ candidate mass to be in between 3.070 and $3.117 \mathrm{GeV} / \mathrm{c}^{2}$ for candidates reconstructed from $\mu^{+} \mu^{-}$, or in between 3.065 and $3.117 \mathrm{GeV} / \mathrm{c}^{2}$ in the case of $e^{+} e^{-}$, a mass-constrained fit is performed. For the $\pi^{+} \pi^{-}$ pair not associated with the $K_{s}^{0}$, the condition $m\left(\pi^{+} \pi^{-}\right)-m\left(J / \psi \pi^{+} \pi^{-}\right)+m_{J / \psi}>-0.150 \mathrm{GeV} / \mathrm{c}^{2}$ is applied, where $m_{J / \psi}$ is the known $J / \psi$ mass [14], in order to reduce combinatorial backgrounds and misidentified $\gamma \rightarrow e^{+} e^{-}$conversions. To reduce the $e^{+} e^{-} \rightarrow q \bar{q}(q=u, d, s, c)$ background events, a selection on the normalized Fox-Wolfram moments $R_{2}$ is applied [15]. The $B$ meson candidates are selected with the energy difference $\Delta E=E_{B}^{\mathrm{cms}}-\sqrt{s} / 2$ and the beam-constrained mass $M_{b c}=\sqrt{s / 4-\left(p_{B}^{\mathrm{cms}}\right)^{2}}$, where $E_{B}^{\mathrm{cms}}$ and $p_{B}^{\mathrm{cms}}$ are the energy and momentum of the $B$ candidate in the center-of-mass system, respectively. The signal candidates are required to have $M_{b c}>5.27 \mathrm{GeV} / \mathrm{c}^{2}$ and $\Delta E<0.02 \mathrm{GeV}$. The $\mathrm{m}\left(J / \psi \pi^{+} \pi^{-}\right)$distribution is used to extract the signal. A histogram probability density function (PDF) generated with signal monte-carlo (MC) samples assuming the known mass of $X(3872)$ [9] and the width from the LHCb measurement [11] is used for the signal component. A straight line is used as a PDF for the background component. A simultaneous fit is performed so as to combine the distribution for $B^{0}$ decay and that for $B^{+}$decay, where the ratio of the expected signal yields is constrained to $\mathcal{B}\left(B^{0} \rightarrow X(3872) K^{0}\right) / \mathcal{B}\left(B^{+} \rightarrow X(3872) K^{+}\right)=$ $0.50 \pm 0.14$ (stat) [12].

Belle II experiment reconstructed in early data a X(3872) signal of $14.4 \pm 4.6$ decays, corresponding to a statistical significance of 4.6 standard deviations as shown in Figure 2. This study is validated with a control sample $B \rightarrow \psi(2 S) K$ as shown in Figure 3. Here, three gaussian functions with a common mean are used as a PDF for the signal component, where all parameters except the mean and scaling factor of the sigmas are determined with signal MC samples. A straight line is used as a PDF for the background component.
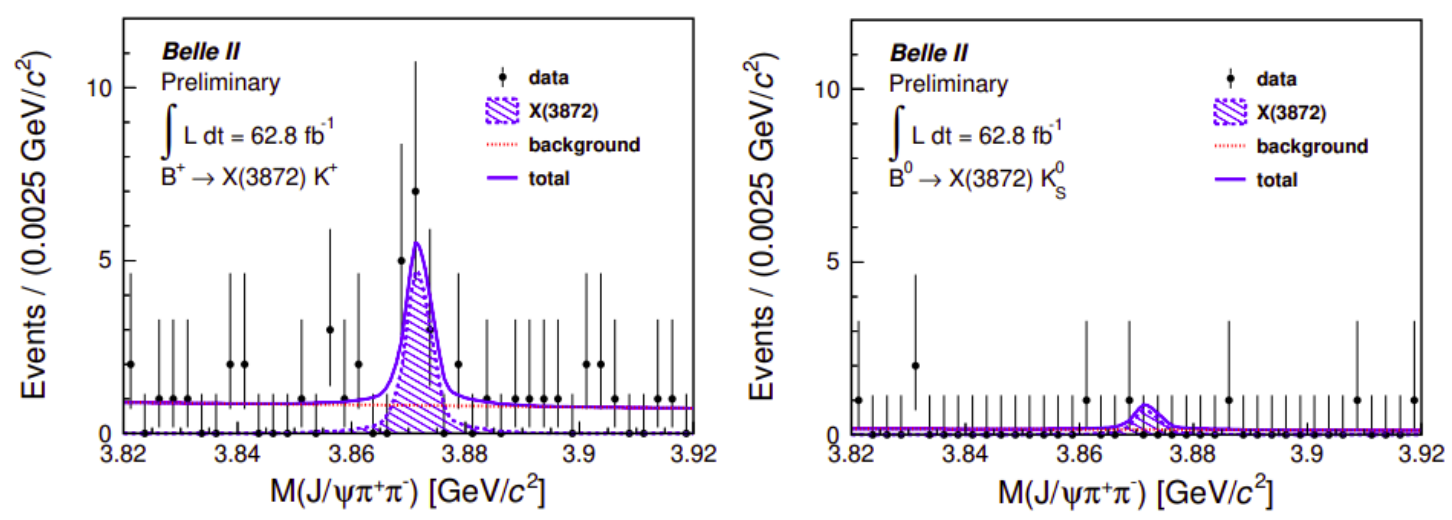

Figure 2: Distributions of $\mathrm{m}\left(J / \psi \pi^{+} \pi^{-}\right)$of $X(3872)$ candidates from (left) $B^{+} \rightarrow J / \psi \pi^{+} \pi^{-} K^{+}$and (right) $B^{0} \rightarrow J / \psi \pi^{+} \pi^{-} K_{S}^{0}$ decays reconstructed in Belle II data. The blue line shows the projection of the total fit. 

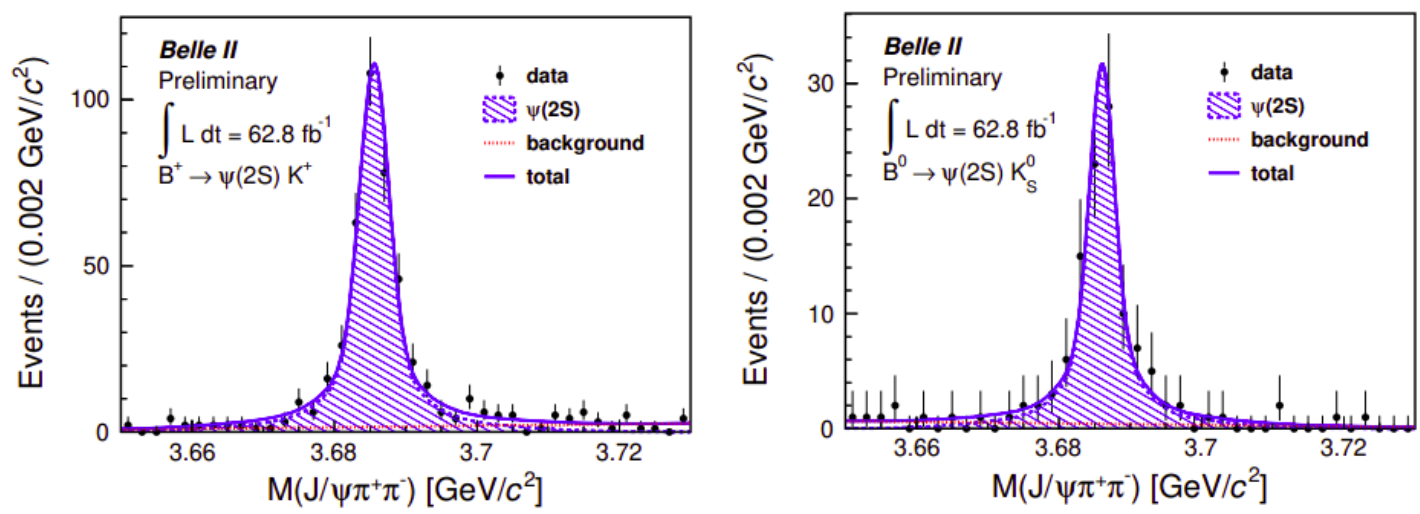

Figure 3: Distributions of $\mathrm{m}\left(J / \psi \pi^{+} \pi^{-}\right)$of $\psi(2 S)$ candidates from (left) $B^{+} \rightarrow \psi(2 S) K^{+}$and (right) $B^{0} \rightarrow \psi(2 S) K_{s}^{0}$ decays reconstructed in Belle II data. The blue line shows the projection of the total fit.

To understand the complete nature of $\mathrm{X}(3872)$, it is necessary to measure its natural width, for which only an upper limit of $1.2 \mathrm{MeV}$ (at the $90 \%$ confidence level) [8] has been obtained with $J / \psi \pi^{+} \pi^{-}$decay. The Belle II measurement of the natural width is planned with $B \rightarrow K X(3872)$, $X(3872) \rightarrow D D \pi$ decays which offer a low Q-value, and therefore improved mass resolution over the $J / \psi \pi^{+} \pi^{-}$channel. Figure 4 shows that $3 \sigma$ sensitivity on the width is expected to be accessible with $10 \mathrm{ab}^{-1}$ or more data.

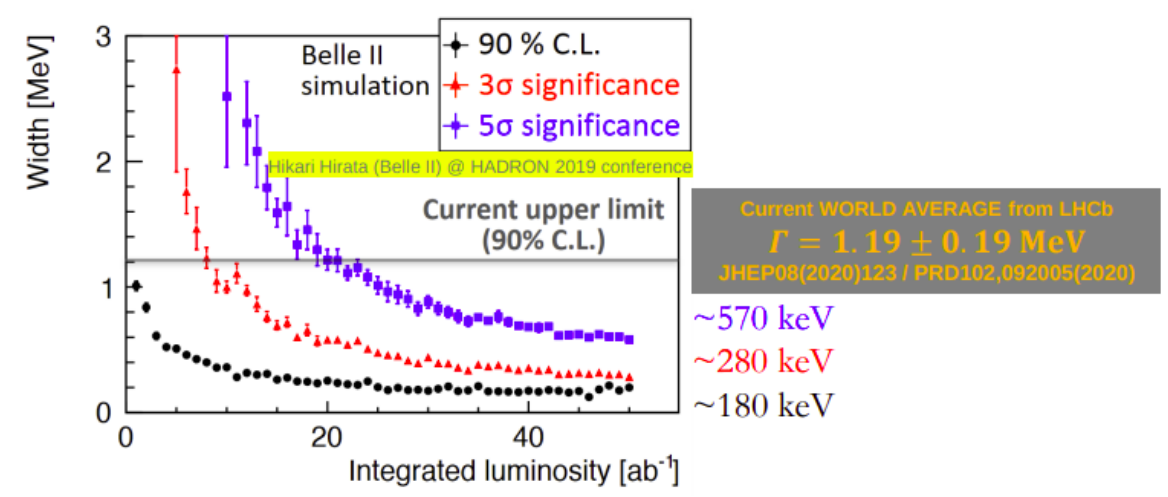

Figure 4: Sensitivity on the width of the $X(3872)$ as a function of the expected integrated luminosity at Belle II. The plot shows MC simulations of the estimated upper limit at $90 \%$ C.L. (black dots), at $3 \sigma$ (red dots) and at $5 \sigma$ (blue dots) of the measured $X(3872)$ width at Belle II. The horizontal black line is fixed to the current upper limit of $1.2 \mathrm{MeV}$ for comparison [13].

\section{ISR results at Belle II}

ISR is a useful physics phenomenon to study $J^{P C}=1^{--}$states below the center-of-mass energy. The advantage of ISR is that the whole hadron spectrum is visible so that the line shape of the resonance and fine structures can be investigated. 
The decay $e^{+} e^{-} \rightarrow \pi^{+} \pi^{-} J / \psi$ via ISR is studied using data corresponding to $37.8 \mathrm{fb}^{-1}$ collected at the $\Upsilon(4 \mathrm{~S})$ resonance. The $J / \psi$ meson is reconstructed from $e^{+} e^{-}$or $\mu^{+} \mu^{-}$pairs within a mass range of $75 \mathrm{MeV} / \mathrm{c}^{2}$ from the known value. An upper bound on the squared value of the missing momentum (due to ISR) in the $J / \psi \pi \pi$ system is imposed at $2 \mathrm{GeV} / c^{2}$. A clear signal of ISR $\psi(2 S)$ is seen as shown in Figure 5.
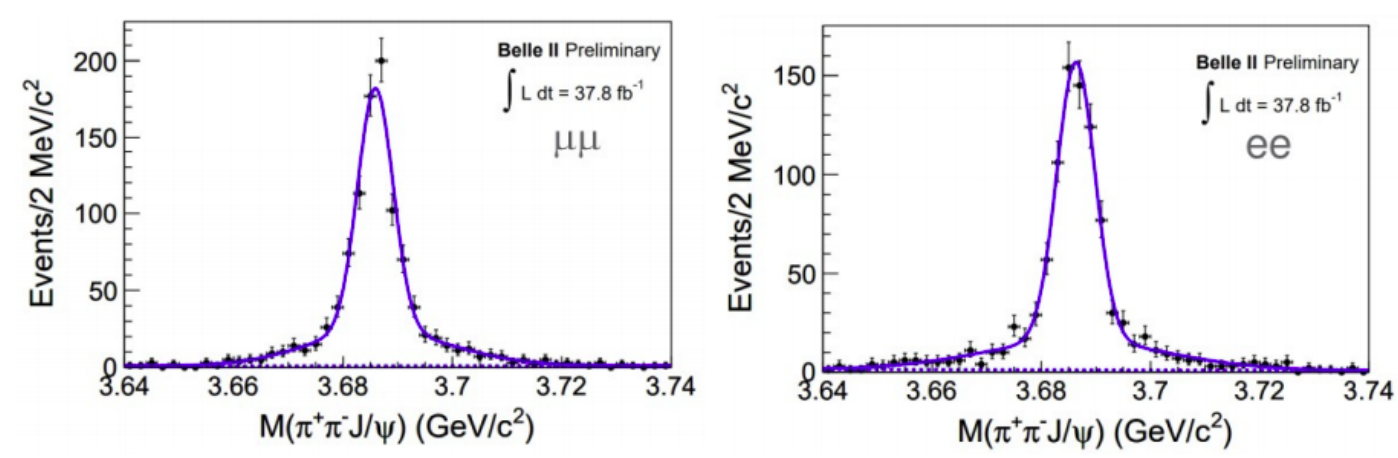

Figure 5: Invariant $J / \psi \pi^{+} \pi^{-}$mass distributions for the candidates reconstructed in the ISR process $e^{+} e^{-} \rightarrow$ $\pi^{+} \pi^{-} J / \psi$. The blue line shows the projection of the total fit.

\section{Summary}

The Belle II experiment is already performing well and plans to accumulate a data set similar to Belle. Studies of quarkonium and XYZ states is one of the keystones of the physics program. With the significant increase of sample size compared to Belle, Belle II can improve some of the measurements already performed by Belle, and look for new charmonium-like states. Belle II can measure more precisely the lineshapes of the states, determine their spin-parities and search for new decay channels. One of the unique aspects of the XYZ program at Belle II is the ability to study $Z$ states in both $B$ meson decays and in direct production via ISR. It is expected that Belle II will make a significant impact on exotic quarkonium spectroscopy.

\section{References}

[1] M. Gell-Mann and E.P. Rosenbaum "Elementary Particles". Scientific American , 72-86 (1957).

[2] G. Zweig "An SU3 Model for Strong Interaction Symmetry and its Breaking”. CERN Report 8419/TH.401 (1964).

[3] S.-K. Choi et al. (Belle Collaboration), Phys. Rev. Lett. 91, 262001 (2003).

[4] D. Acosta et al. (CDF Collaboration), Phys. Rev. Lett. 93, 072001 (2004).

[5] V.M. Abazov et al. (DO Collaboration), Phys. Rev. Lett. 93, 162002 (2004).

[6] B. Aubert et al. (BaBar Collaboration), Phys. Rev. D 71, 071103 (2005). 
[7] R. Aaij et al. (LHCb Collaboration), Eur Phys. J. C 72, 1972 (2012).

[8] R. Aaij et al. (LHCb Collaboration), Phys. Rev. D 102, 092005 (2020)

[9] T.Abe et al. (Belle Collaboration), KEK Report 2010-1 (2010).

[10] M. Tanabashi et al. (Particle Data Group), Phys. Rev. D 98, 030001 (2018).

[11] R. Aaij et al. (LHCb Collaboration), J. High Energ. Phys. 2020, 123.

[12] S.-K. Choi et al. (Belle Collaboration), Phys. Rev. D 84, 052004 (2011).

[13] H. Hirata (2019, August 16-21), Sensitivity to the X(3872) total width at the Belle II experiment, 18th International Conference on Hadron Spectroscopy and Structure (HADRON 2019): Guilin, Guangxi, People's Republic of China.

[14] P. A. Zyla et al. (Particle Data Group), Prog. Theor. Exp. Phys. (2020) 083 C01 and 2021 update.

[15] G. C. Fox and S. Wolfram, Phys. Rev. Lett. 41, 1581 (1978). 